\title{
Molecular Surveillance of Antimalarial Resistance Pfcrt, Pfmdr1 and Pfk13 Polymorphisms in African Plasmodium Falciparum Imported Parasites to Wuhan, China
}

\section{Cheng Weijia}

Hubei University of Medicine

Xiaonan Song

Hubei University of Medicine

Huabing Tan

Hubei University of Medicine

Kai Wu

Wuhan Centers for Disease Prevention and Control

Jian Li ( $\square$ yxlijian@163.com )

Hubei University of Medicine https://orcid.org/0000-0001-9434-7803

\section{Research}

Keywords: Plasmodium falciparum, pfk13, pfcrt

Posted Date: October 9th, 2020

DOI: https://doi.org/10.21203/rs.3.rs-87992/v1

License: (c) (1) This work is licensed under a Creative Commons Attribution 4.0 International License.

Read Full License 


\section{Abstract}

Background: The development of drug resistance in Plasmodium falciparum becomes a severe problem for malaria control globally. Before finding a practical solution, monitoring the susceptibility of $P$. falciparum resistance-related genes is crucial. It will offer valuable information on the drug resistance in malaria-endemic areas and guides the rational clinical use of antimalarial drugs.

Methods冈Filter paper blood was taken from patients with positive $P$. falciparum during 2017-2019 in Wuhan, China. The target fragments from $p f c r t, p f m d r 1$, and $k 13$ propeller (pfk13) genes of $P$. falciparum were amplified and sequenced. Subsequently, the polymorphisms of $p f c r t, p f m d r 1$, and $p f k 13$ and the haplotypes of Pfcrt and Pfmdr1 were analyzed.

Results: Totally, 106 samples were collected. Subsequently, 98.11\% (104/106), 100\% (106/106), and $86.79 \%(92 / 106)$ of these samples were successfully amplified and sequenced for the $p f c r t, p f m d r 1$, and pfk13 genes, respectively. The prevalence of Pfcrt K76T, Pfmdr1 N86Y, and Pfmdr1 Y184F mutation were $9.62 \%, 4.72 \%$, and $47.17 \%$, respectively. At codons $72-76$ of pfort gene locus were showed three haplotypes, including CVMNK (wild-type), CVIET (mutation type), CV M/I N/E K/T (mixed type), with $87.50 \%, 9.62 \%$, and $2.88 \%$ prevalence, respectively. For the $p f m d r 1$ gene, including NY (wild type), NF and YF (mutant type), N Y/F, Y Y/F, and N/Y Y/F (mixed type), accounted for $34.91 \%, 43.40 \%, 3.77 \%, 15.09 \%$, $0.94 \%$, and $1.89 \%$, respectively. A total of eleven Pfcrt/Pfmdr1 combined haplotypes, including six types of combined haplotypes, and five combined haplotypes with mixed-type, For pfk13, no mutation was detected.

Conclusions: The wild-type SNPs and haplotypes for the $p f c r t$, and $p f m d r 1$ genes become predominant in the current study. It indicates these isolates entirely or partly recovery their susceptibility to antimalarial drugs, including chloroquine, amodiaquine, and mefloquine. Moreover, it demonstrates these drugs can continue to be effective drugs for $P$. falciparum malaria cases treatment in Africa. Although no mutation is detected in $p f k 13$, continuous molecular surveillance is still urgently necessary.

\section{Introduction}

Malaria is a mosquito-borne infectious disease that seriously endangers human health, among which falciparum malaria caused by Plasmodium falciparum is the most serious, mainly in tropical and subtropical regions, especially in sub-Saharan Africa and Southeast Asia (SEA) [1]. According to the latest WHO estimates in 2019, during 2018, there were 228 million new malaria cases worldwide and about 405,000 deaths from malaria [1]. Research demonstrates that since 2017, China has achieved zero reporting of indigenous malaria cases for three consecutive years [2]. In recent years, with the process of globalization, the number of migrant workers, tourists, and businessmen in China is increasing gradually, especially those returning from Africa and SEA [3], which has brought serious pressure for malaria control in China. It has brought severe challenges to the imported malaria control work of our country and even the whole world. 
At present, drug therapy was the primary means for prevention and treatment of malaria [4]. However, with the use of increasingly antimalarial and anti-mosquito drugs, P. falciparum and its vector Anopheles mosquitoes gradually develop drug resistance and spread rapidly [5]. Chloroquine (CQ) is a safe, cheap, and effective antimalarial drug for malaria treatment. However, in the 1940s, $P$. falciparum parasites developed resistant to $\mathrm{CQ}$. Since then, CQ-resistant (CQR) strains began to spread rapidly around the world [6]. After the discovery of artemisinin (ART) in the 1970s, malaria control was temporarily eased. Unfortunately, ART-resistant strains have been reported in SEA, which has raised global awareness of malaria treatment and management [7]. In our previous study, an $\mathrm{R} 561 \mathrm{H}$ mutation of $p f k 13$ was found in a sample from Rwanda, Africa, in 2016 [8]. A recent study found the mutation was independent occurred in Africa [9], confirming our previous findings. In order to achieve better therapeutic results, artemisininbased combination therapy (ACTs) was recommended by WHO as the most extensive and useful program for malaria treatment. As the core component of ACTs or other adjuvant combination drugs produces widespread drug resistance, the incidence of malaria treatment failure will be high. Therefore, in the absence of more choices, it is urgent to monitor drug resistance genes related to major antimalarial drugs.

At present, studies have confirmed that gene mutations such as $p f k 13, p f c r t$, and $p f m d r 1$ have been used as the primary molecular markers of drug resistance in malaria parasites [7, 10-12]. The $p f k 13$ polymorphism has been considered to be related to ART resistance. However, studies have shown that the distribution of alleles for $p f k 13$ varies according to the mutation [13]. In SEA, the alleles of C580Y mutation was dominant [13]. In Africa, the mutation rate of the $p f k 13$ remains relatively low level. Recently, the newly discovered local drug resistance mutation site in Africa-R561 H $[8,9,14]$. The $72-76$ amino acid mutation in Pfcrt, especially the K76T mutation, was the primary marker of CQR $[10,15]$. Several mutations in pfmdr1 will cause the resistance of $P$. falciparum to CQ, amodiaquine (AQ) and mefloquine (MQ), and other drugs $[11,16]$.

In the current study, pfcrt, pfmdr1, and pfk13 polymorphisms of $P$. falciparum isolates imported from Africa in Wuhan City, Hubei Province, China was surveyed. It will provide the gene mutation and the related data of $P$. falciparum resistance detection, and offer significant clues to prevent the spread of drug-resistant $P$. falciparum in Africa, and China.

\section{Materials And Methods}

\subsection{Collection of samples}

From May 2017 to December 2019, $3 \mathrm{ml}$ of peripheral blood of returned migrant workers from Africa were collected in Wuhan, Hubei Province. These samples were examined by immune-gold assay kit (ICT Diagnostics) for Plasmodium spp. antigen (HRPII). Then, blood smears with patient samples were prepared and checked under microscopic. Finally, the species were identified by qPCR. Approximately $400 \mu$ l of blood was spotted on 3 MM Whatman filter paper and air-dried (identified and provided by the Center for Disease Control and Prevention of Wuhan City, Hubei Province). Then, these filter papers were 
numbered and stored at $-20^{\circ} \mathrm{C}$ with a polyethylene bag. The consent of the owner and his legal guardian has been obtained before sampling.

\subsection{Determination of $P$. falciparum gene mutations}

Genomic DNA (gDNA) was extracted from blood-spots by TIANamp Blood Spots DNA Kit, to yield approximately $50 \mu \mathrm{l}$ of supernatant containing DNA and stored $-20^{\circ} \mathrm{C}$ until further use. Using the extracted gDNA as a template, the target fragments of Pfcrt gene (Gene ID: PF3D7_0709000), pfmdr1 gene (Gene ID: PF3D7_0523000), and pfk13 gene (Gene ID: PF3D7_1343700). Following the previously published primer information and following the previous procedure $[8,17,18]$ to modify slightly and establish PCR reaction, the $p f c r t, p f m d r 1$, and $p f k 13$ genes were successfully amplified. The reaction system and reaction procedure for PCR were shown in Table 1. After all the reactions were finished, the $5 \mu \mathrm{l}$ second-round PCR products were analyzed by $1.0 \%$ agarose gel electrophoresis. Then the remaining products were purified for sequenced in Genewiz, Soochow, China. The sequencing data were analyzed by Dnastar (DNASTAR Inc., Madison, WI, USA), and compared with Pfcrt, Pfmdr1, and pfk 13 of $P$. falciparum 3D7 strain as a standard sequence. The new mutation was verified by a new PCR product and bi-directional DNA sequencing.

\subsection{Data analysis}

Analyze the data using SPSS 25 (SPSS Inc., Chicago, IL, USA). The allele frequencies and $95 \%$ confidence intervals of wild-type and mutant types for these genes were calculated [8].

\section{Results}

\subsection{General information}

A total of 151 individuals were diagnosed with malaria from 2017 to 2019 . Of these, 106 were infected with P. falciparum, 17 with Plasmodium vivax, 20 with Plasmodium ovale, and 8 with Plasmodium vivax. Among 106 cases of $P$. falciparum infection, all from African (Table S1), including 48 cases in West Africa, 33 cases in Central Africa, 15 cases in South Africa, 9 cases in East Africa, and 1 case in North Africa. These cases were mainly from 21 African countries, dominating distributed in Congo $(21.70 \%$, $23 / 106)$, followed by Nigeria $(16.98 \%, 18 / 106)$, Ivory Coast $(9.43 \%, 10 / 106)$, Mozambique $(6.60 \%, 7 / 106)$. No cases have been found in SEA.

\subsection{Mutation prevalence of Pfcrt and Pfmdr1}

The gDNA was extracted from 106 samples of $P$. falciparum patients. Then, PCR amplification was performed, and all positive PCR products were obtained. For pfcrt, a total of 104 samples were successfully sequenced $(98.11 \%, 104 / 106)$. The prevalence of Pfcrt V73V, M74I, N75E, K76T allele mutation was $9.62 \%$ (10/104) in Africa (Table 2). These mutations were mainly concentrated in West Africa, followed by Central Africa. And the results exhibited that the pfcrt genotype had polymorphisms at codons 72-76, including CVMNK (wild-type), CVIET (mutation type), and CV M/I N/E K/T (mixed type). 
Most isolates harboured parasites with the CVIET (87.5\%, 91/104). The proportion of CVIET among these isolates was $9.62 \%$ (10/104). The last case was CV M/I N/E K/T, accounting for $2.88 \%$ (3/104), no SVMNT haplotype was found. (Table S2).

For pfmdr1 gene, $100 \%$ samples were obtained the PCR products, and 100\% PCR products were sequenced successfully (Table 2). Sequencing results showed the main epidemic mutation sites of pfmdr1 were concentrated on N86Y and Y184F, with $4.72 \%$ and $47.17 \%$ mutation, respectively (Table 2). These mutations were also mainly focused in West Africa, followed by Central Africa and South Africa. In addition, six haplotypes encoding N86Y and Y184F were detected (Table S2), including NY (wild type), NF and YF (mutant type), N Y/F, Y Y/F, and N/Y Y/F (mixed type), accounted for $34.91 \%, 43.40 \%, 3.77 \%$, $15.09 \%, 0.94 \%$, and $1.89 \%$, respectively. Besides, one non-synonymous mutation at position 199 and several synonymous mutations at position 87 and 102 were detected in these samples (Table 3 and Fig. 1).

With these polymorphisms in pfcrt and pfmdr1 genes, a total of eleven Pfcrt/Pfmdr1 combined haplotypes were assessed. There were six types of combined haplotypes, namely NF-CVIET, NF-CVMNK, NY-CVIET, NY-CVMNK, YF-CVIET, YF-CVMNK. They were mainly concentrated in West Africa and Central Africa. Among them, Congo and Nigeria accounted for $16.98 \%$ (18/106) and 13.21\% (14/106) respectively. The other five combined haplotypes with mixed-type, including N Y/F-CV M/I N/E K/T, N Y/F -CVMNK, N/Y Y/F-CVMNK, NF-CV M/I N/E K/T, Y Y/F-CVMNK. Also concentrated in West Africa and Central Africa, mainly distributed in Ivory Coast 5.66\% (6/106) and Congo 4.72\% (5/106).

\subsection{Analysis of mutation in Pfk13 gene}

For the $p f k 13,93.40 \%$ (99/106) samples were amplified, and 92.93\% (92/106) cases were sequenced successfully. Sequencing analysis displayed that these cases were all wild-type, and no mutation was detected at positions $474,476,493,508,527,533,537,539,543,553,568,574,578$, and 580 of the Pfk 13 gene. The previously detected mutations at positions 550, 561, 575, and 589 in Africa were also not detected.

\section{Discussion}

For decades, the rapid emergence of drug resistance of $P$. falciparum parasites has become one of the leading causes of the global burden for malaria [19]. The continuous influx of imported malaria infections in China increases the possibility of malaria re-spreading to all acceptable areas in China [20]. Therefore, we investigated the $p f k 13, p f c r t$, and $p f m d r 1$ polymorphisms of imported $P$. falciparum resistance genes from Africa in Wuhan City, Hubei Province, China. According to the results, at codons 72-76 of pfcrt gene locus were showed three haplotypes, including CVMNK, CVIET, and CV M/I N/E K/T, which were $87.50 \%, 9.62 \%$, and $2.88 \%$, respectively. For Pfmdr1 gene, the NY (34.91\%) and NF (43.40\%) were the most popular haplotypes. For the $p f k 13$, both the mutations detected in SEA [21] and novel mutations detected in Africa [9] were not found in the current study. These results deliver information on 
the mutations of $P f c r t, P f m d r 1$, and $p f k 13$ genes of imported $P$. falciparum isolate in Wuhan and thus provide valuable information for the status of drug-resistant $P$. falciparum in China.

Africa and SEA were the primary sources of imported malaria in Wuhan [8]. According to this survey, among the three haplotypes of the Pfcrt gene, CVMNK, CVIET and CV M/I N/E K/T demonstrates that the pressure on CQ drugs decreased significantly after most countries in sub-Saharan Africa stopped using $\mathrm{CQ}$ in the 1990s [22-25]. Compared with the previous investigation of four haplotypes of Pfcrt [8], the prevalence rates of CVMNK, SVMNT and CVIET, and CV M/I N/E K/T were 50.57\%, 1.14\%, 25.00\%, and $23.30 \%$, respectively. The relative increase in CVMNK genotypes of Pfcrt was also reported in Senegal and southeastern Cameroon [26, 27], which confirmed that the proportion of CQ-susceptibility parasites in Africa is increasing after drug withdrawal, which was remarkable phenomenon, but it is still not recommended to quote $\mathrm{CQ}$ at this time. Current national use and regulation of antimalarial drugs should continue to be implemented to prevent the recurrence of CQR development.

Previously reported N86Y, Y184F allele mutations in Pfmdr1 are most prevalent in Asia and Africa [28]. In this study, we monitored the situation of $\mathrm{N} 86 \mathrm{Y}$ and $\mathrm{Y} 184 \mathrm{~F}$ in Africa, accounting for $4.72 \%$ and $47.17 \%$. Compared with the previous survey [8], the prevalence rates of N86Y (22.28\%) and Y184F (60.01\%), $\mathrm{Y} 184 \mathrm{~F}$ allele mutation still dominates. It has been confirmed that N86Y mutation was near related to CQR and AQ resistance $[29,30]$. The prevalence rates of N86Y dropped significantly from $22.28 \%$ in the previous survey [8] in 2011-2016 to $4.72 \%$ in this survey from 2017 to 2019 , indicating that susceptibility to $C Q$ and $A Q$ drugs was recovering. In recent years, several studies demonstrate the N86Y, Y184F, and D1246Y were closely related to artemether-lumefantrine $(A L)$ resistance [31, 32]. The prevalence rates of Y184F increased from $22.28 \%$ in the previous survey in $2011-2016$ to $47.17 \%$ in this survey from 2017 to 2019, which remained at a high level. Also, we found a non-synonymous mutation at position 199 and synonymous mutations, including 87 and 102.

For the $p f k 13$, no mutation was found in this survey, which may be a worthy symbol that there was still a high level of sensitivity to ART in Africa, and drugs can continue to be used. In our previous study, a mutation of R561 H was reported in Rwanda [8]. It has also been reported in another study [14]. Recently, it was identified the mutation has ART resistance [9]. All these pieces of evidence indicate that ART resistance of $P$. falciparum strain was independent produced in Africa and not spread from SEA. The phenomenon needs to pay more attention to China and Africa. If there are no effective measures to curb this situation, then the general resistance of $C Q$ is likely to be the end of ART.

However, there are also several limitations to the present study. First, because of the COVID-19 outbreak in 2019, especially in these low-income tropical countries [33,34], these may hinder malaria control efforts, leading to a shortage of data. Second, some of the data were missing because of the failure of amplification or sequencing.

\section{Conclusions}


All in all, the wild-type SNPs and haplotypes for the pfcrt and pfmdr1 genes of $P$. falciparum become predominant in the current study. It indicates that these isolates entirely or partly recovery their susceptibility to antimalarial drugs, including CQ, AQ, and MQ. Moreover, it demonstrates these drugs can continue to be effective drugs for $P$. falciparum malaria cases treatment in Africa. Although no mutation is detected in $p f k 13$, cautions should be made to ART therapy for $P$. falciparum in Africa, continuous molecular surveillance is still urgently necessary.

\section{Declarations}

\section{Competing interests}

The authors declare that they have no competing interests.

\section{Authors' contributions}

WJC and JL conceived and designed the study. KW coordinated the field collections of patient isolates. KW carried out a microscopic examination. WJC and XNS performed the experiments. WJC, HBT, and JL analyzed the data. WJC and JL wrote the paper. All the authors read and approved the final manuscript.

\section{Acknowledgments}

The authors would like to thank the Department of Schistosomiasis and Endemic Diseases, Wuhan Center for Disease Prevention and Control, and to all participants who have contributed their blood samples.

\section{Availability of data and material}

The datasets analyzed in this study are available from the corresponding author on reasonable request.

\section{Consent for publication}

Not applicable

\section{Ethics approval and consent to participate}

The current study was approved by the ethics committees of the Hubei University of Medicine and Wuhan City Center for Disease Prevention and Control Ethics Committee. Informed consent was obtained from all participated individuals.

\section{Funding}

This study was supported by the Foundation for Innovative Research Team of Hubei University of Medicine (Grant Number YC2020003) and the National Natural Science Foundation of China (Grant Number 81802046). 


\section{References}

1. WHO: World malaria report 2019.Geneva:World Health Organization.

2. Feng J, Zhang L, Huang F, Yin JH, Tu H, Xia ZG, Zhou SS, Xiao N, Zhou XN: Ready for malaria elimination: zero indigenous case reported in the People's Republic of China.Malar J 2018, 17:315.

3. Feng J, Xiao H, Zhang L, Yan H, Feng X, Fang W, Xia Z: The Plasmodium vivax in China: decreased in local cases but increased imported cases from Southeast Asia and Africa.Sci Rep 2015, 5:8847.

4. Antony HA, Parija SC: Antimalarial drug resistance: An overview.Trop Parasitol 2016, 6:30-41.

5. Achan J, Mwesigwa J, Edwin CP, D'Alessandro U: Malaria medicines to address drug resistance and support malaria elimination efforts.Expert Rev Clin Pharmacol 2018, 11:61-70.

6. Payne D: Spread of chloroquine resistance in Plasmodium falciparum.Parasitol Today 1987, 3:241246.

7. Ashley EA, Dhorda M, Fairhurst RM, Amaratunga C, Lim P, Suon S, Sreng S, Anderson JM, Mao S, Sam B, et al: Spread of artemisinin resistance in Plasmodium falciparum malaria.N Engl J Med 2014, 371:411-423.

8. Yao Y, Wu K, Xu M, Yang Y, Zhang Y, Yang W, Shang R, Du W, Tan H, Chen J, et al: Surveillance of Genetic Variations Associated with Antimalarial Resistance of Plasmodium falciparum Isolates from Returned Migrant Workers in Wuhan, Central China.Antimicrob Agents Chemother 2018, 62.

9. Uwimana A, Legrand E, Stokes BH, Ndikumana JM, Warsame M, Umulisa N, Ngamije D, Munyaneza T, Mazarati JB, Munguti K, et al: Emergence and clonal expansion of in vitro artemisinin-resistant Plasmodium falciparum kelch13 R561H mutant parasites in Rwanda.Nat Med 2020.

10. Djimde A, Doumbo OK, Cortese JF, Kayentao K, Doumbo S, Diourte Y, Coulibaly D, Dicko A, Su XZ, Nomura T, et al: A molecular marker for chloroquine-resistant falciparum malaria.N Engl J Med 2001, 344:257-263.

11. Reed MB, Saliba KJ, Caruana SR, Kirk K, Cowman AF: Pgh1 modulates sensitivity and resistance to multiple antimalarials in Plasmodium falciparum.Nature 2000, 403:906-909.

12. Ariey F, Witkowski B, Amaratunga C, Beghain J, Langlois AC, Khim N, Kim S, Duru V, Bouchier C, Ma L, et al: A molecular marker of artemisinin-resistant Plasmodium falciparum malaria.Nature 2014, 505:50-55.

13. Bonnington CA, Phyo AP, Ashley EA, Imwong M, Sriprawat K, Parker DM, Proux S, White NJ, Nosten F: Plasmodium falciparum Kelch 13 mutations and treatment response in patients in Hpa-Pun District, Northern Kayin State, Myanmar.Malar J 2017, 16:480.

14. Wang X, Ruan W, Zhou S, Huang F, Lu Q, Feng X, Yan H: Molecular surveillance of Pfcrt and k13 propeller polymorphisms of imported Plasmodium falciparum cases to Zhejiang Province, China between 2016 and 2018.Malar J 2020, 19:59.

15. Cooper RA, Ferdig MT, Su XZ, Ursos LM, Mu J, Nomura T, Fujioka H, Fidock DA, Roepe PD, Wellems TE: Alternative mutations at position $\mathbf{7 6}$ of the vacuolar transmembrane protein PfCRT are 
associated with chloroquine resistance and unique stereospecific quinine and quinidine responses in Plasmodium falciparum.Mol Pharmacol 2002, 61:35-42.

16. Eyase FL, Akala HM, Ingasia L, Cheruiyot A, Omondi A, Okudo C, Juma D, Yeda R, Andagalu B, Wanja $\mathrm{E}$, et al: The role of Pfmdr1 and Pfcrt in changing chloroquine, amodiaquine, mefloquine and lumefantrine susceptibility in western-Kenya P. falciparum samples during 2008-2011.PLoS One 2013, 8:e64299.

17. Li J, Chen J, Xie D, Eyi UM, Matesa RA, Ondo Obono MM, Ehapo CS, Yang L, Yang H, Lin M: Limited artemisinin resistance-associated polymorphisms in Plasmodium falciparum K13-propeller and PfATPase6 gene isolated from Bioko Island, Equatorial Guinea.Int J Parasitol Drugs Drug Resist 2016, 6:54-59.

18. Li J, Chen J, Xie D, Eyi UM, Matesa RA, Obono MMO, Ehapo CS, Yang L, Yang H, Lin M, et al: Molecular mutation profile of Pfcrt and Pfmdr1 in Plasmodium falciparum isolates from Bioko Island, Equatorial Guinea.Infect Genet Evol 2015, 36:552-556.

19. Cibulskis RE, Alonso P, Aponte J, Aregawi M, Barrette A, Bergeron L, Fergus CA, Knox T, Lynch M, Patouillard E, et al: Malaria: Global progress 2000 - 2015 and future challenges.Infect Dis Poverty 2016, 5:61.

20. Zhou S, Li Z, Cotter C, Zheng C, Zhang Q, Li H, Zhou S, Zhou X, Yu H, Yang W: Trends of imported malaria in China 2010-2014: analysis of surveillance data.Malar J 2016, 15:39.

21. Talundzic E, Okoth SA, Congpuong K, Plucinski MM, Morton L, Goldman IF, Kachur PS, Wongsrichanalai C, Satimai W, Barnwell JW, Udhayakumar V: Selection and spread of artemisininresistant alleles in Thailand prior to the global artemisinin resistance containment campaign.PLOS Pathog 2015, 11:e1004789.

22. Kublin JG, Cortese JF, Njunju EM, Mukadam RA, Wirima JJ, Kazembe PN, Djimde AA, Kouriba B, Taylor TE, Plowe CV: Reemergence of chloroquine-sensitive Plasmodium falciparum malaria after cessation of chloroquine use in Malawi.J Infect Dis 2003, 187:1870-1875.

23. Shretta R, Omumbo J, Rapuoda B, Snow RW: Using evidence to change antimalarial drug policy in Kenya.Trop Med Int Health 2000, 5:755-764.

24. Eriksen J, Nsimba SE, Minzi OM, Sanga AJ, Petzold M, Gustafsson LL, Warsame MY, Tomson G: Adoption of the new antimalarial drug policy in Tanzania-a cross-sectional study in the community.Trop Med Int Health 2005, 10:1038-1046.

25. Kamya MR, Bakyaita NN, Talisuna AO, Were WM, Staedke SG: Increasing antimalarial drug resistance in Uganda and revision of the national drug policy.Trop Med Int Health 2002, 7:1031-1041.

26. Ndiaye M, Faye B, Tine R, Ndiaye JL, Lo A, Abiola A, Dieng Y, Ndiaye D, Hallett R, Alifrangis M, Gaye O: Assessment of the molecular marker of Plasmodium falciparum chloroquine resistance (Pfcrt) in Senegal after several years of chloroquine withdrawal.Am J Trop Med Hyg 2012, 87:640-645.

27. Ndam NT, Basco LK, Ngane VF, Ayouba A, Ngolle EM, Deloron P, Peeters M, Tahar R: Reemergence of chloroquine-sensitive pfcrt K76 Plasmodium falciparum genotype in southeastern Cameroon.Malar J 2017, 16:130. 
28. Veiga MI, Dhingra SK, Henrich PP, Straimer J, Gnadig N, Uhlemann AC, Martin RE, Lehane AM, Fidock DA: Globally prevalent PfMDR1 mutations modulate Plasmodium falciparum susceptibility to artemisinin-based combination therapies.Nat Commun 2016, 7:11553.

29. Duraisingh MT, Drakeley CJ, Muller O, Bailey R, Snounou G, Targett GA, Greenwood BM, Warhurst DC: Evidence for selection for the tyrosine-86 allele of the pfmdr 1 gene of Plasmodium falciparum by chloroquine and amodiaquine.Parasitology 1997, 114 ( Pt 3):205-211.

30. Folarin OA, Bustamante C, Gbotosho GO, Sowunmi A, Zalis MG, Oduola AM, Happi CT: In vitro amodiaquine resistance and its association with mutations in pfcrt and pfmdr1 genes of Plasmodium falciparum isolates from Nigeria.Acta Trop 2011, 120:224-230.

31. Baliraine FN, Rosenthal PJ: Prolonged selection of pfmdr1 polymorphisms after treatment of falciparum malaria with artemether-lumefantrine in Uganda.J Infect Dis 2011, 204:1120-1124.

32. Happi CT, Gbotosho GO, Folarin OA, Sowunmi A, Hudson T, O'Neil M, Milhous W, Wirth DF, Oduola AM: Selection of Plasmodium falciparum multidrug resistance gene 1 alleles in asexual stages and gametocytes by artemether-lumefantrine in Nigerian children with uncomplicated falciparum malaria.Antimicrob Agents Chemother 2009, 53:888-895.

33. Nghochuzie NN, Olwal CO, Udoakang AJ, Amenga-Etego LN, Amambua-Ngwa A: Pausing the Fight Against Malaria to Combat the COVID-19 Pandemic in Africa: Is the Future of Malaria Bleak? Front Microbio/ 2020, 11:1476.

34. Chanda-Kapata P, Kapata N, Zumla A: COVID-19 and malaria: A symptom screening challenge for malaria endemic countries.Int J Infect Dis 2020, 94:151-153.

35. Ishengoma DS, Saidi Q, Sibley CH, Roper C, Alifrangis M: Deployment and utilization of nextgeneration sequencing of Plasmodium falciparum to guide anti-malarial drug policy decisions in sub-Saharan Africa: opportunities and challenges.Malar J 2019, 18:267.

\section{Tables}

Due to technical limitations, table $1,2,3$ is only available as a download in the Supplemental Files section.

\section{Figures}




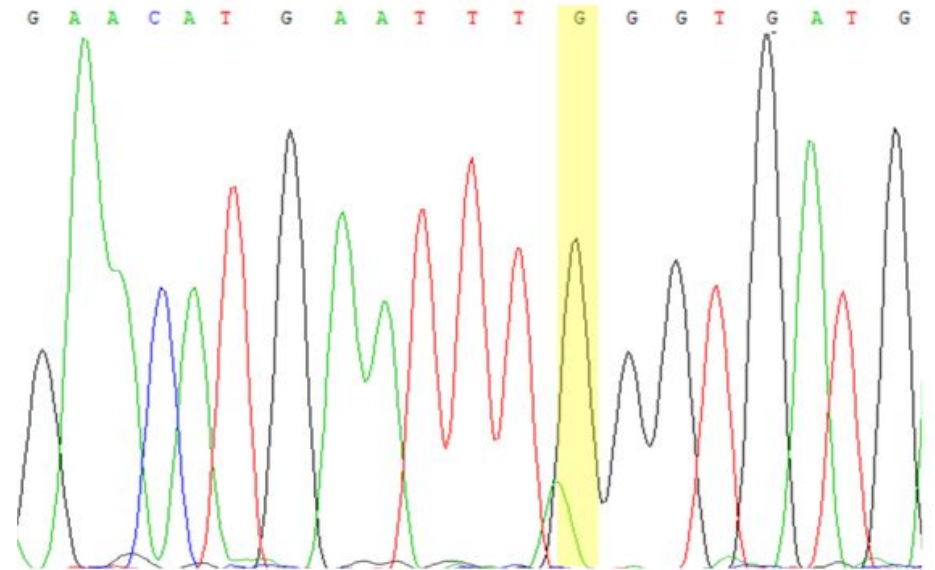

A261G (T87T)

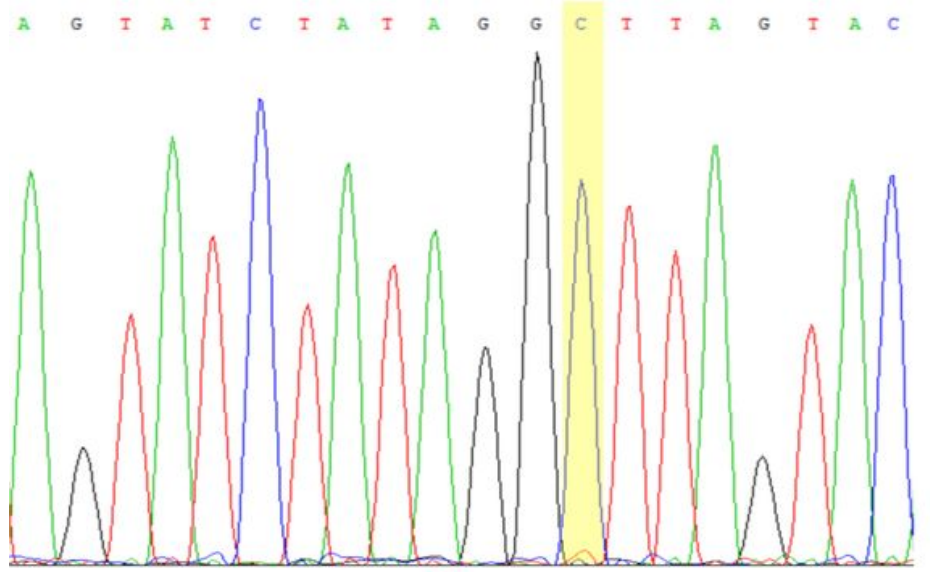

T306C (G102G)

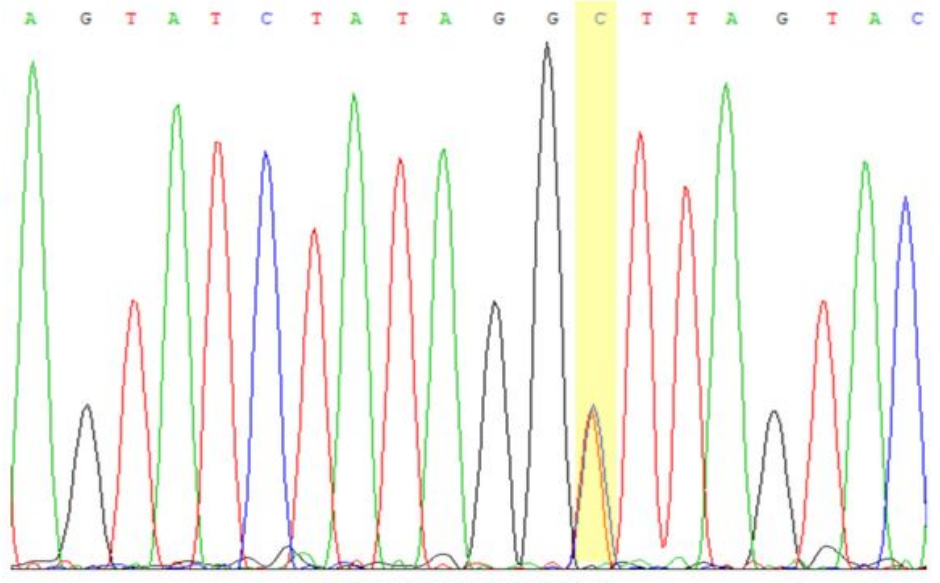

$\mathrm{T} 306 \mathrm{~T} / \mathrm{C}(\mathrm{G} 102 \mathrm{G} / \mathrm{T})$

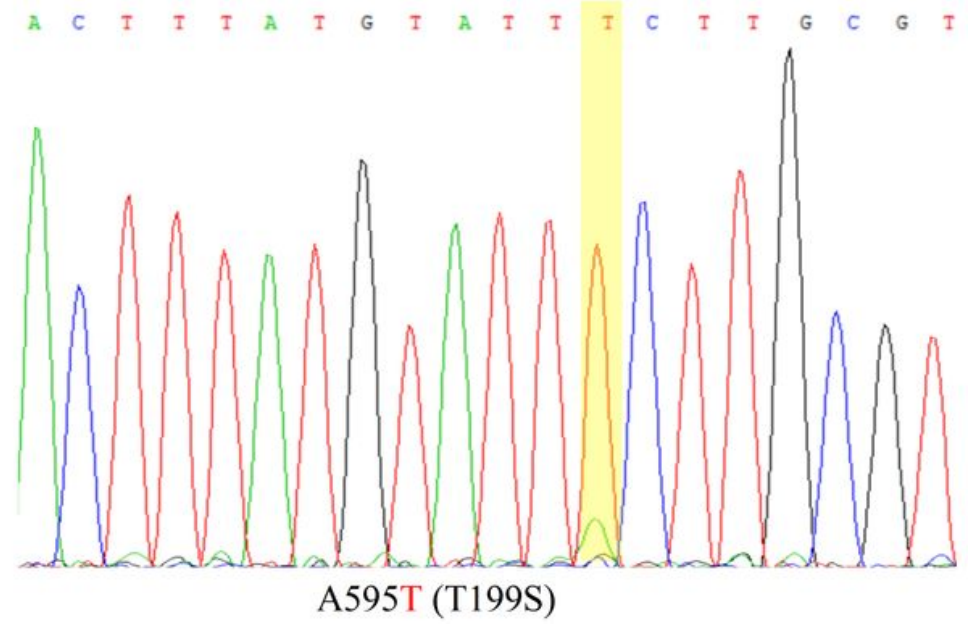

\section{Figure 1}

Sequence profile of PCR products of the new mutation site of pfmdr1 genes.

\section{Supplementary Files}

This is a list of supplementary files associated with this preprint. Click to download.

- Table2.xlsx

- Table3.xlsx

- Table1.xlsx

- TableS2.xIsx

- TableS1.xIsx 